\title{
Gray-Space Spectrum Sharing between Multiple Rotating Radars and Cellular Network Hotspots
}

\author{
Rathapon Saruthirathanaworakun***, Jon M. Peha*, and Luis M. Correia** \\ * Carnegie Mellon University, PA, USA, ** IST/INOV-INESC - Tech. Univ. Lisbon, PORTUGAL \\ e-mail: rsaruthi@alumni.cmu.edu,peha@cmu.edu, luis.correia@inov.pt
}

\begin{abstract}
This paper considers gray-space spectrum sharing when rotating radars are primary spectrum users, and multiple cells from one or more cellular networks are secondary users. A cellular network may share spectrum to supplement its dedicated spectrum, or provide a broadband hotspot service. A secondary device is allowed to transmit as long as cumulative interference is not harmful to nearby radars, probably because no radar is pointing its directional antenna at the device at this moment. This paper presents mechanisms that would support such sharing, and quantifies performance when spectrum is considered $100 \%$ utilized under traditional spectrum management. It is shown that the sharing allows cells to sustain significant mean data rates. For example, if $5 \%$ of a cellular network's cells need more capacity than dedicated spectrum can provide, a cell can get almost $1.2 \mathrm{bps} / \mathrm{Hz}$ on average from shared spectrum. By evaluating quality of service, it is found that shared spectrum could be used efficiently for applications such as non-interactive video streaming, peer-to-peer file sharing, large file transfers, and web browsing, but not for applications such as real-time transfers of small files, and VoIP.
\end{abstract}

Keywords- Primary-Secondary Spectrum Sharing; Opportunistic; Gray-Space; Multiple Rotating Radars; Cellular

\section{INTRODUCTION}

As regulators struggle to find spectrum for new purposes such as broadband [1], sharing spectrum with radar becomes increasingly important because radar operates in so much of the spectrum. In the US, $1.1 \mathrm{GHz}$ of spectrum from 0.225 to $3.7 \mathrm{GHz}$ is used by non-military, fixed land-based radars [2].

We previously evaluated the overall extent of transmissions achievable by considering one cell of a cellular system sharing spectrum with one radar [2]-[4]. An opportunistic primarysecondary sharing model [5] was considered; a secondary spectrum user can transmit only when it will not cause harmful interference to a primary system. Harmful interference is interference resulting in noticeable disruption of service [6].

We extend the sharing model in [2]-[4] to when multiple cells share the same frequency band with multiple radars. These active cells do not blanket the region, e.g., a cellular system provides broadband hotspots, or only uses shared spectrum when a temporary surge of traffic requires more capacity than what is available from its dedicated spectrum.

Existing models and proposals to share spectrum with radars are usually based on white-space sharing, in which secondary transmissions are allowed in a region where the spectrum is detected as "unused". The existing model in the
$5 \mathrm{GHz}$ band is based on Dynamic Frequency Selection (DFS), allowing unlicensed devices to transmit when no radar signals are detected; a similar idea was investigated in the $2.8 \mathrm{GHz}$ band [7], [8]. In other bands such as the $3.5 \mathrm{GHz}$ band, NTIA has proposed allowing non-radar systems to operate except in "exclusion zones," which are conservatively calculated such that radars and secondary devices cannot harm each other [9].

In contrast, this paper and [2]-[4] consider more efficient spectrum sharing, i.e., gray-space sharing [10], through which a secondary device is allowed to transmit near a radar, but only at a power that will not cause harmful interference. In this paper, the max allowable transmit power of a secondary device is dynamically controlled according to the state of the radar.

This paper considers the most typical radar of which the main beam rotates (a rotating radar), e.g., Air Traffic Control (ATC) and weather radars. With the rotation, gain of the radar antenna seen by a fixed secondary device varies over time. Hence, link loss (including antenna gains and path loss) between the device and the radar is occasionally high enough that the device can transmit successfully without causing harmful interference, although with interruptions and fluctuations. This sharing can be achieved either with or without explicit communications between the secondary and the primary systems, i.e., cooperative or coexistent sharing [5].

[11] qualitatively discussed the idea of sharing spectrum with rotating radar. This paper and our previous work [2]-[4] were the first to quantify the extent of transmissions achievable, and the impact of interruptions and fluctuations in transmissions on the performance of prominent applications on the Internet. [12] found that access opportunities for an unlicensed device could be increased using information on varying link loss between a radar and the device.

We propose mechanisms to control transmissions of each cell so that the cumulative interference will not be harmful to any radar. Only downstream cellular transmissions are considered; the upstream will be addressed in future work. An Internet service provider may use this shared spectrum only for downstream, in part because downstream Internet traffic greatly exceeds upstream traffic, and because in spectrum shared with rotating radar, downstream was found to be more spectrally efficient [2]-[4].

The sharing scenarios and mechanisms are explained in Sections II and III, respectively. Performance measures of the sharing are described in Section IV. Numerical results are presented in Section V, and conclusions are in Section VI.

Support for this research was provided by the Fundação para a Ciência e a Tecnologia (Portuguese Foundation for Science and Technology) through the Carnegie Mellon Portugal Program under Grant SFRH/BD/33594/2008. 


\section{SHARING SCENARIOS}

The cellular system considered is OFDMA-based. Secondary transmissions occur simultaneously in some but not all cells around the radars.

A radar uses the same antenna for transmission and reception. The radiation pattern of the radar, as seen by a secondary device, depends on the angle between the main beam and the device. The radar transmits a series of pulses with constant power, and detects echoes of the pulses from its surroundings [2]. Rotation speed and main beam direction of a radar need not be the same as the other radars'.

To protect radars from harmful interference, the Interference-to-Noise Ratio (INR) must be kept below an established value with sufficiently high probability. To achieve this, a secondary device can adjust its transmissions according to a radar's changing antenna gain, as long as the device has some information on current state of the main beam. It is assumed that a secondary device knows either i) current antenna gain of a radar together with the expected path loss between itself and the radar or ii) the summation of the gain and path loss, and that the device can remain synchronized as the radar's antenna gain changes. The device can determine this loss in various ways, depending on the sharing approach and type of radar. For example, consider an ATC radar, horizontally rotating at a constant speed. In coexistent sharing, a secondary device may combine any a priori knowledge of the radar with observations over numerous rotations to determine the periodic pattern of the expected link loss. Alternatively, some observations can be replaced by direct communications in cooperative sharing [5]. For radars that occasionally change direction or speed, e.g., weather radars reacting when a storm approaches, cooperative sharing should be used.

The sharing model assumes: 1) some technical information of a radar- such as tolerable interference level, pulse power, and rotating period- is known to the secondary system, which can be encouraged through spectrum policy [10] (These parameters rarely change over time.); 2) the cellular system: a) will use as much available bandwidth as possible, and b) can always transmit signaling traffic without harming a radar. (This could easily happen, e.g., if signaling is transmitted in a frequency band different from the one shared with radars.)

This paper considers a specific case in which inter-cell interference among cells is negligible. As secondary transmissions occur in some but not all cells, it is unlikely that all neighboring cells will interfere with each other as occurs in a typical cellular system. Moreover, interference among neighboring cells can be reduced further by mechanisms such as those used in LTE to mitigate inter-cell interference.

\section{Controlling Cellular Systems to Prevent HARMFUL INTERFERENCE}

Transmissions of each of multiple Base Stations (BSs) need to be controlled, such that the total interference is not harmful. A BS can determine when it can transmit and at what power using two complementary mechanisms.

Regional resource allocation allocates the shared spectrum resource to each active cell, possibly using information from across the region, such as the probability that each BS will be active, and link loss between BSs and radars. These allocations do not change as radars rotate. In addition, each BS has a local power control that adjusts its maximum transmit power based only on local information, so adjustments can be made quickly, and without coordination among cells.

Of the multiple ways to do this, the regional resource allocation used in this paper specifies a threshold on link loss $\left(L_{T, j}\right)$ between each radar $j$ and its nearby BSs. The local power control allows a BS to transmit at the equipment power limit $P_{\max }$ when the expected link loss is below this threshold, and no transmissions otherwise. This expected link loss can differ from its instantaneous value due to, e.g., fading. The threshold does not change as the radar rotates, but changes with the fraction of active BSs, or if there were a substantial shift in the geographic clustering of active cells.

BS $i$ will transmit when its expected link loss to each of its nearby radars is below the associated threshold. At any current main beam direction of each of the radars nearby, represented here as vector $\vec{\varphi}$, transmit power of the $\mathrm{BS}, P_{B S, i}(\vec{\varphi})$, is

$P_{B S, i}(\vec{\varphi})=\left\{\begin{array}{cc}P_{\max } & , \text { if } \forall j, \bar{L}_{i, j}\left(\varphi_{j}\right)<L_{T, j} \\ 0 & \text {, otherwise }\end{array}\right.$.

$\bar{L}_{i, j}\left(\varphi_{j}\right)$ is the expected link loss between nearby radar $j$ and BS $i$; the main beam direction of radar $j$ is $\varphi_{j} . \bar{L}_{i, j}$ accounts for the radar's antenna gain $G_{R d, j}\left(\varphi_{j}\right)$, the BS antenna gain, and expected path loss between the radar and the BS.

The threshold is determined such that the risk of harmful cumulative interference to any of the associated radars is sufficiently low. Even without change in the fraction of active cells, and hence no change in expected cumulative interference, the instantaneous cumulative interference varies due to, e.g., 1) fading and shadowing, 2) which specific cells are active, and 3) whether each cell has enough data to send with maximum power. The threshold will exceed the expected value enough to protect the radars from these fluctuations, and is derived from the distribution of this cumulative interference, which can be obtained analytically, empirically, or by simulation.

\section{PERFORMANCE MEASUREMENT}

Performance of the sharing will be evaluated from achievable data rate, fraction of time that a cell is allowed to transmit, and the interruptions and fluctuation in secondary transmissions as radars rotate.

The data rate, achieved by cell $i$ is calculated as the mean data rate averaged across the cell area $E_{c e l l}\left\{R_{b, i}(\vec{\varphi})\right\}$. The data rate $R_{b, i}(\vec{\varphi})$ is a function of $\operatorname{SINR} \rho_{i}(\vec{\varphi})$, which depends on the current direction $\vec{\varphi}$ of the $N_{R d, i}$ radars nearby. Given that inter-cell interference among active cells is negligible, $E_{c e l l}\left\{R_{b, i}(\vec{\varphi})\right\}$ is calculated using these additional assumptions: i) the SINR of secondary transmissions is conservatively calculated without considering time between radar pulses, which is very small [13]; ii) the location of users is uniformly distributed throughout each cell, and as often occurs with LTElike systems, each user gets an equal share of spectrum; and iii) the data rate is approximated as a fraction $\gamma$ of Shannon's limit, 
to roughly approximate what can be observed on an LTE-like system. With the transmit power of BS $i$ (i.e., $\left.P_{B S, i}\right)$, in (1),

$\rho_{i}(\vec{\varphi})=\frac{L_{S, i}\left(d_{S}\right) \times P_{B S, i}(\vec{\varphi})}{n_{S} \times W_{S}+\sum_{j=1}^{N_{R d, i}}\left[L_{R d, j-M T}\left(\varphi_{j}\right) \times P_{R d, j}\right]}$.

$W_{S}$ is the bandwidth of secondary transmissions, and $L_{S, i}\left(d_{S}\right)$ is link loss between BS $i$ and its Mobile Terminals (MTs) $d_{S}$ away. $n_{S}$ is background noise power spectral density at the MTs. $P_{R d, j}$ is transmit power of radar $j$, and $L_{R d, j-M T}\left(\varphi_{j}\right)$ is instantaneous link loss between the radar and the MTs.

Because secondary transmissions can be interrupted, a user, at a given location in a cell, will experience different perceived data rates $R_{b, s, p}\left(t_{s t}\right)$ when transferring files of different sizes. $R_{b, S, p}$ is defined as the ratio of file size to total file transfer time $T_{f} ; T_{f}$ depends on the start time $t_{s t}$. By choosing different $t_{s t}$ values, the distributions of $T_{f}$ and $R_{b, S, p}$ can be obtained.

To study the fluctuations in $R_{b, S, p}$, we consider collocated users at a fixed location in cells, located at various distances from a radar. In a cell, the users are at the edge closest to the radar, resulting in the worst-case data rate and fluctuations.

\section{NUMERICAL RESULTS}

\section{A. Assumptions for Numerical Results}

This paper uses Monte Carlo simulation to determine the extent of cellular communications operating in a spectrum band that, by conventional spectrum management, would be considered $100 \%$ utilized by radars. Radars are as densely packed as possible on an infinite plane, without causing harmful interference to each other; white-space sharing is not possible. Each radar is $d_{R d}$ away from its six adjacent radars. This same plane is blanketed by cellular cells of equal size, each of which is active with probability $a$, independent of which other cells are active. $a$ is sufficiently low that inter-cell interference is negligible. The only areas that are not covered by cells are those in the immediate vicinity $d_{\min }$ of radar.

Conservatively, the ITU-R P.1546 model [14] in flat terrain is adopted for path loss from radar to a cell. This increases interference between radar and the cell, and thus reduces transmissions achievable. Path loss from BS to MT follows the Walfisch-Ikegami model [15]. A wireless channel is subjected to unit-mean shadowing and multipath fading. The shadowing and fading are log-normal and Rice distributed, respectively.

The link loss threshold of a given $\operatorname{radar} j\left(L_{T, j}\right)$ is set such that even if every eligible active cell transmits at the equipment power limit, see (1), it is very unlikely that total interference to any radar will exceed the radar's tolerable limit. The thresholds are determined as follows. For a given threshold, the distributions of 1) fraction of time that an active cell is allowed to transmit, 2) interference that an active cell would cause to each of its nearby radars, and 3) maximum data rate achievable by an active cell are determined via simulation. The likelihood that a cell is active is equal in all locations, except within radius $d_{\min }$ from any radar. For a given threshold, the relationship between the probability that total interference will exceed the radar tolerable limit vs. the percentage of cells that can be active $a$ can be determined, using the distribution of interference from an active cell, obtained via simulation. The relationship is determined under the simplifying assumptions that i) all cells are equally likely to be active, and ii) interference from different active cells is independent so the sum of these random variables can be approximated with a normal distribution. Unless stated otherwise, the probability of harmful interference is less than $0.1 \%$ [2]; the values of other parameters used in simulations are summarized in Table I.

TABLE I. PARAmeters USED In Simulations (UnLESS STATED OTHERWISE)

\begin{tabular}{|c|c|}
\hline Parameters & Value \\
\hline $\begin{array}{l}\text { Radar [2][13]: } \\
\text { Operating Frequency (e.g., by ATC radars) [GHz] } \\
\text { Bandwidth [MHz] } \\
\text { Uniformly-Distributed Aperture Antenna }{ }^{1} \\
\text { - Elevation 3-dB Beamwidth [degree] } \\
\text { - Azimuthal 3-dB Beamwidth [degree] } \\
\text { - Main Beam Gain [dBi] } \\
\text { - Front-to-Back Ratio [dB] } \\
\text { - Height [m] } \\
\text { Rotating Period [s] } \\
\text { Transmit Power }\left(P_{R d}\right)[\mathrm{MW}] \\
\text { Interference to Noise Ratio (INR) [dB] } \\
\text { Background Noise [dBm] } \\
\text { Distance between two Radars } d_{R d}[\mathrm{~km}]^{2}\end{array}$ & $\begin{array}{c}4.7 \\
1.4 \\
33.5 \\
38 \\
30 \\
4.7 \\
0.45 \\
-10 \\
-106 \\
280 \\
\end{array}$ \\
\hline $\begin{array}{l}\text { Cellular [2]: } \\
\text { Antenna Gain of a MT (Omni-Directional) }[\mathrm{dBi}] \\
\text { Antenna Gain of a BS (Sectorized) }[\mathrm{dBi}] \\
\text { Cell Radius (assuming an urban or suburban area) }[\mathrm{m}] \\
\text { Equipment Power Limit of a BS }\left(P_{\max }\right)[\mathrm{dBm}] \\
\text { Background Noise Spectral Density }\left(n_{S}\right)[\mathrm{dBm} / \mathrm{Hz}] \\
\text { Noise Figure at a Receiver }[\mathrm{dB}] \\
\text { Minimum Distance to a Radar } d_{\min }[\mathrm{km}]\end{array}$ & $\begin{array}{r}0 \\
18 \\
800 \\
46 \\
-174 \\
5 \\
5\end{array}$ \\
\hline $\begin{array}{l}\text { COST 231 Walfisch-Ikegami Model [15]: } \\
\text { Building Height (small to medium height) [m] } \\
\text { MT Antenna Height }[\mathrm{m}] \\
\text { BS Antenna Height }[\mathrm{m}]^{3}\end{array}$ & $\begin{array}{l}15 \\
1.7 \\
30\end{array}$ \\
\hline $\begin{array}{l}\text { Other Parameters: } \\
\text { Fraction of Shannon's limit } \gamma \text { in Section } \mathrm{IV}^{4} \\
\text { Shadowing Parameter } \sigma_{S h}[\mathrm{~dB}] \\
\text { Rice K Factor }[\mathrm{dB}] \\
\text { - Within Line of Sight }(\mathrm{LoS}) \text { from a Radar } \\
\text { - Beyond LoS from a Radar }\end{array}$ & $\begin{array}{c}0.53 \\
7 \\
10 \\
0\end{array}$ \\
\hline
\end{tabular}

\section{B. Extent of Secondary Transmissions}

The extent of transmissions is measured as the fraction of time that a BS can transmit, and mean data rate achievable. The mean data rate per active cell is $E_{\text {cell }}\left\{R_{b, i}(\vec{\varphi})\right\}$, as defined in Section IV, averaged across all active cells, and all directions of all nearby radars. We also investigate the impact of three parameters on the extent of transmissions: distance between a BS and its nearest radar, percentage of cells that are active, and acceptable risk of harmful interference to radars.

Fig. 1 shows (a) percentage of time that a BS is allowed to transmit, and (b) mean data rate per active cell vs. distance between a BS and its nearest radar, when $4 \%, 12 \%$ or $20 \%$ of

\footnotetext{
${ }^{1}$ The antenna is up-tilted, to reduce reflected signals from the ground, so that its gain in the horizontal direction is $5 \mathrm{~dB}$ lower than the main beam gain [2].

${ }^{2}$ According to the US Federal Aviation Administration (FAA), distance between two radars using the same frequency should be at least $280 \mathrm{~km}$ [16]

${ }^{3}$ With this value, cell radius can be as large as $1.5 \mathrm{~km}$. $30 \mathrm{~m}$ represents a reasonable compromise between an on-tower antenna and a rooftop one [2].

${ }^{4}$ This results in minimum mean square error between the estimated data rate and the data rate obtained from 3GPP data regressions [2].

${ }_{5}^{5}$ The LoS distance between a radar and a BS considered is $20.8 \mathrm{~km}$, see [2].
} 
cells are active. The results are from the worst-case scenario in which white-space sharing is not possible. Interference caused to radars by the active cells is at maximum; hence, protecting radars from harmful interference has to be very stringent.

Fig. 1 shows that distance between a BS and a radar is one important factor affecting the extent of transmissions achievable. The transmissions are low when a BS is very close to a radar (e.g., at $10 \mathrm{~km}$ away), but quite high when the BS is tens of km away. Even in this extremely limited scenario, the proposed sharing scheme makes extensive secondary transmissions possible in an area where white-space sharing is not possible. At $100 \mathrm{~km}$ from the nearest radar, a BS can transmit $28 \%$ of the time when the percentages of cells active is $4 \%$. The resulting mean data rate per active cell is $28 \%$ of that achievable in dedicated spectrum (system rate limit), which is around $3.6 \mathrm{bps} / \mathrm{Hz}$ [2]. Even with $20 \%$ of cells active, $0.54 \mathrm{bps} / \mathrm{Hz}$ data rate is high enough to support mediumquality (1.6 Mbps) video streaming [2] in just $3 \mathrm{MHz}$ of shared spectrum. Interruptions during transmissions would not be a problem as long as 3 to 4 s, i.e., less than $1 \mathrm{MB}$, of content can be buffered because typical rotating period of the radar is only around $4.7 \mathrm{~s}$ [13].

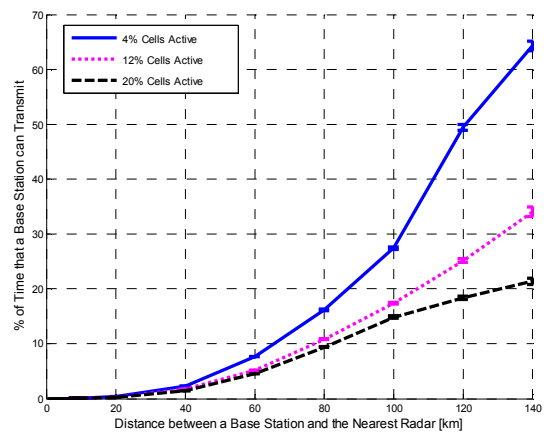

(a) Percentage of Time that a Base Station can Transmit

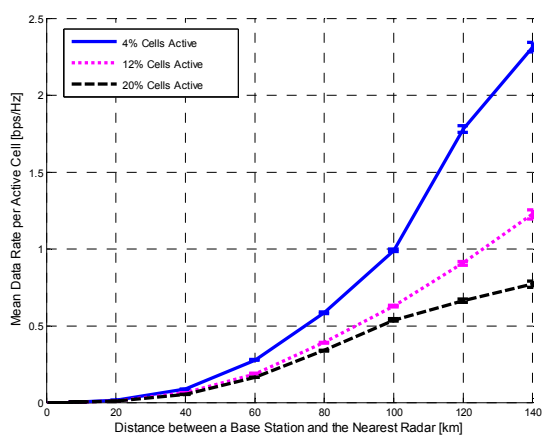

(b) Mean Downstream Data Rate per Active Cell

Figure 1. Extent of secondary transmissions with $95 \%$ confidence interval vs. Distance between a base station and its nearest radar

Fig. 2 shows mean data rate per cell, averaged across all active cells, vs. percentage of cells active $(a)$. The risk of harmful interference to radar is either $0.1 \%$ or $0.5 \%$. Although increasing $a$ would reduce the achievable data rate per active cell, it increases total system throughput. For example, at $0.1 \%$ risk of harmful interference, increasing $a$ from $4 \%$ to $12 \%$, reduces the achievable mean data rate per cell by less than a factor of two while tripling the number of active cells. Thus, even though the data rate per active cell goes down, the total benefit actually goes up when many cells are active. Hence, the sharing can be useful regardless of whether $a$ at any given time is large or small. Moreover, there is a tradeoff: system designers can choose to achieve high total system throughput by allowing high $a$, or to guarantee a certain level of transmissions of each cell by limiting $a$.

Fig. 2 also shows that the level of risk of harmful interference to a radar has a small impact on the extent that a BS can transmit. Thus, it is possible to provide high protection to radars with little reduction in achievable transmissions.

\section{Fluctuations in Perceived Data Rate and Implications for Application Performance}

Mean data rate is a good performance measure for some applications but not all. We study fluctuations in perceived data rate (defined in Section IV) experienced by a given user, and whether these fluctuations will be a problem for prominent Internet applications including file transfers, Peer-to-Peer file sharing (P2P), web browsing, and Voice-over-IP (VoIP) [2].

Fig. 3 shows the 1 st percentile perceived data rate vs. mean perceived data rate, for files of different size ranging from $10 \mathrm{kB}$ to $10 \mathrm{MB}$. Transferring a small file is more susceptible to high data rate fluctuations. More than $99 \%$ of the time a user downloading files larger than $1 \mathrm{MB}$ would perceive data rate close to the mean. However, a user downloading small files, e.g., $10 \mathrm{kB}$ files, might perceive data rate that is an order of magnitude lower than the mean even when the mean is high; this is spectrally inefficient. The fluctuations make the sharing attractive for transferring of files large enough that the fluctuations are not noticeable, such as song and video downloads. The sharing is also attractive for applications that can tolerate interruptions such as P2P. However, it will be problematic for interactive exchanges of small pieces of data that must be received within a small period.

Although it involves transferring small files, web browsing can be supported with acceptable Quality of Service (QoS), and high spectral efficiency. File transfer time is the important performance measure for web browsing. It has been suggested that the webpage downloading time should not exceed $4 \mathrm{~s}$ [2]. As shown by Fig. 3, this is achieved $99 \%$ of the time with only $3 \mathrm{MHz}$ of shared spectrum for a webpage not larger than $1 \mathrm{MB}$. (Note that $1 \mathrm{MB}$ is large for a webpage, given that the 90th percentile webpage size in 2010 was $660 \mathrm{kB}$ [2].) Even though a user transferring large files will experience less fluctuations in data rate, downloading webpages larger than $1 \mathrm{MB}$ will obviously experience longer downloading time; however, that would be fairly close to what achievable in dedicated spectrum.

In contrast, meeting VoIP QoS requirements is only possible at very low spectral efficiency, even in the simpler case when a cell must only concern itself with one radar [2]. Consider a constant data rate VoIP that requires a latency less than $150 \mathrm{~ms}$. It was found [2] that the required latency could not be met unless the constant data rate is below $0.005 \mathrm{bps} / \mathrm{Hz}$. 
To summarize, even with the fluctuations in data rate, the majority of wireless Internet traffic- including video streaming, large file download, web browsing, and downstream P2P- will work well in shared spectrum in an area where white-space sharing is not possible.

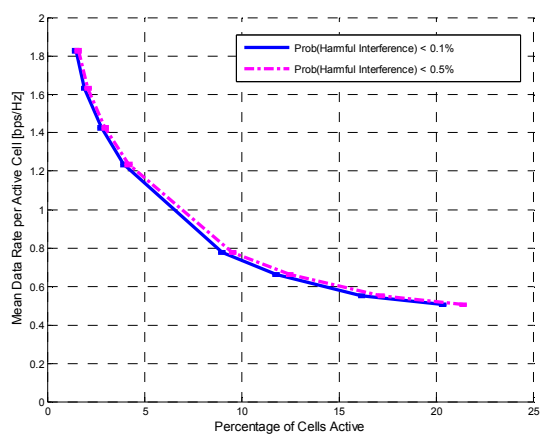

Figure 2. Mean downstream data rate per cell, averaged across all active cells, with $95 \%$ confidence interval vs. Percentage of cells active

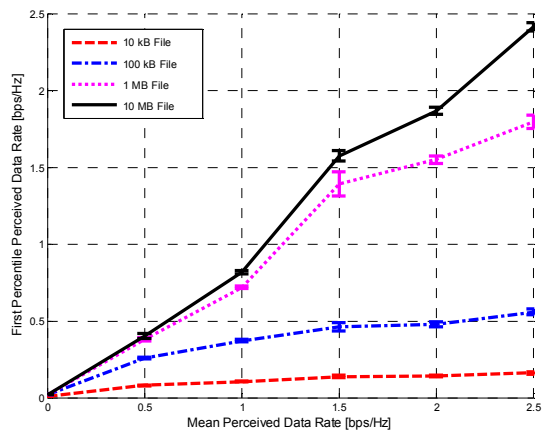

Figure 3. The first percentile perceived data rate with $95 \%$ confidence interval vs. Mean perceived data rate

\section{CONCLUSIONS}

This paper quantifies the potential of opportunistic grayspace sharing between multiple rotating radars and broadband cells. The active cells at a given time do not entirely blanket a region; this might be valuable for a cellular network that uses shared spectrum only when demand exceeds the capacity available in dedicated spectrum, or provides a hotspot service.

We propose that such sharing should be provided using a two-step mechanism: the particular regional resource allocation mechanism considered provides every active cell with a threshold for expected signal loss, and the local power control mechanism ceases transmission during those periods when current expected signal loss falls below that threshold.

The paper deliberately considers the worst possible deployment scenario. Not only is spectrum $100 \%$ utilized as viewed by traditional spectrum management, but radars are also packed to the theoretical maximum. However, sufficiently high data rates can be achieved on average. For example, if instantaneous load exceeds the capacity of dedicated spectrum in $5 \%$ of its cells, a cellular cell can get almost $1.2 \mathrm{bps} / \mathrm{Hz}$ on average from the shared spectrum. Even greater total throughput is possible when more cells are active, although the achievable data rate per cell would be lower. Although dedicated spectrum can support data rates higher than $1.2 \mathrm{bps} / \mathrm{Hz}$, this is impressive from spectrum that is already so heavily utilized. Moreover, this can be done with an interference risk to radar that is likely to be well below the interference risk that these rotating radars already pose to each other, in part because cellular performance was found to be relatively insensitive to the level of risk to radars.

The ability to achieve a significant data rate over spectrum that is inaccessible by white-space sharing is encouraging. Even at high mean data rates, sporadic interruptions in transmission may be problematic for some applications, most notably VoIP and urgent transfers of small files. However, it is found that high levels of fluctuations and interruptions are still tolerable for other applications such as video streaming, web browsing, peer to peer file sharing, and large file transfers which collectively account for most wireless Internet traffic.

\section{REFERENCES}

[1] US Federal Communications Commission (FCC), National Broadband Plan, Mar. 2010

[2] R. Saruthirathanaworakun, J.M. Peha and L.M. Correia, "Opportunistic sharing between rotating radar and cellular," IEEE J. on Selected Area in Comm., vol. 30, no. 10, Nov 2012, pp.1900-10.

[3] J.M. Peha, "Cellular systems and rotating radar using the same spectrum," Proc. of ISART, Boulder, CO, USA, July 2011.*

[4] R. Saruthirathanaworakun, J.M. Peha and L.M. Correia, "Opportunistic primary-secondary spectrum sharing with a rotating radar," Proc. of IEEE ICNC, Maui, HI, USA, Feb. 2012."

[5] J.M. Peha, "Sharing spectrum through spectrum policy reform and cognitive radio," Proc. of the IEEE, vol.97, no.4, Apr. 2009, pp.708-19."

[6] R.P. Margie, "Efficiency, predictability, and the need for an improved interference standard at the FCC," Proc. of 31st Telecommun. Policy Research Conf. (TPRC), Arlington, VA, USA, Sep. 2003.

[7] L. Wang, J. McGeehan, C. Williams, and A. Doufexi, "Radar spectrum opportunities for cognitive communications transmission," Proc. of CrownCom, Singapore, May 2008.

[8] M.I. Rahman and J.S. Karlsson, "Feasibility evaluations for secondary LTE usage in 2.7-2.9 GHz radar bands," Proc. of IEEE PIMRC, Toronto, Canada, Sep. 2011.

[9] US National Telecomm. and Information Administration (NTIA), An assessment of the near-term viability of accommodation wireless broadband systems in the $1675-1710 \mathrm{MHz}, 1755-1780 \mathrm{MHz}, 3500-3$ $650 \mathrm{MHz}$, and 4 200-4 220 MHz, 4 380-4 $400 \mathrm{MHz}$ bands, Oct. 2010.

[10] J.M. Peha, "Spectrum sharing in the gray space," Telecommunications Policy Journal, In Press, Available Online: Aug. 2012."

[11] M.J. Marcus, "Sharing government spectrum with private users: opportunities and challenges," IEEE Wireless Communications Magazine, vol. 16, no. 3, June 2009, pp. 4-5.

[12] M. Tercero, K.W. Sung, and J. Zander, "Temporal secondary access opportunities for WLAN in radar bands," Proc. of WPMC, Brest, France, Oct. 2011.

[13] ITU-R, Characteristics of radiolocation radars, and characteristics and protection criteria for sharing studies for aeronautical radionavigation and meteorological radars in the radiodetermination service operating in the frequency band 2 700-2 $900 \mathrm{MHz}, \mathrm{M} .1464-1,2003$.

[14] ITU-R, Method for point-to-area predictions for terrestrials services in the frequency range $30 \mathrm{MHz}$ to $3000 \mathrm{MHz}, \mathrm{P} .1546-4,2009$.

[15] E. Damosso and L.M. Correia (eds.), COST 231 Final Report - Digital Mobile Radio: Evolution Towards Future Generation Systems, COST Secretariat, European Commission, Brussels, Belgium, 1999.

[16] US Federal Aviation Administration (FAA), Radar Quick Reference Guide, V2.0, AJW-63, ATC Spectrum Engineering Service, July 2007.

\$ www.ece.cmu.edu/ peha/wireless.html 